This is an author-produced electronic version of an article accepted for publication in the British Journal of Psychiatry. The definitive publisherauthenticated version is available online at http://bjp.rcpsych.org. 


\title{
The CIDI-Core Substance Abuse and Dependence Questions: Cross-cultural and Nosological Issues
}

\author{
L. B. COTTLER, L. N. ROBINS, B. F. GRANT, J. BLAINE, L. H. TOWLE, H.-U. \\ WITTCHEN, N. SARTORIUS \\ and PARTICIPANTS IN THE WHO/ADAMHA FIELD TRIAL
}

The CIDI is a fully standardised, structured interview for the assessment of psychiatric disorders according to DSM-II-R and proposed ICD-10 criteria. The development of this interview has been the collaborative effort of researchers from 18 sites around the world. In a field trial to test the crosscultural acceptability and reliability of the questions, there was found to be high acceptance and excellent reliability for the substance use questions, problems with the lengthy alcohol section, and difficulties translating relevant substance use concepts into different languages. There is therefore room for further improvement in the substance-related questions. There proved to be differences between ICD-10 and DSM-III-R regarding substance abuse and dependence disorders.

The Composite International Diagnostic Interview (CIDI) Core Version 1.0 (World Health Organization, 1987) is a fully standardised diagnostic interview for the assessment of psychiatric disorders which allows their classification according to DSM-III-R criteria (American Psychiatric Association, 1987) and ICD-10 (World Health Organization, 1989a). The collaborative efforts to develop this instrument have been made possible by the World Health Organization (WHO) and the US Alcohol, Drug Abuse, and Mental Health Administration (ADAMHA) Joint Project on Diagnosis and Classification of Mental Disorders, and Alcohol and Drug-Related Problems. This is one product of the efforts initiated in 1980when the WHO/ADAMHA Joint Project began to support the development of a family of instruments for psychiatric assessment.

The CIDI is an epidemiological instrument that allows non-clinician interviewers to ask questions in a highly standardised fashion, and to elicit answers which can be combined by computer to produce diagnoses according to the published criteria of different diagnostic systems. The CIDI Core Version 1.0 (hereafter referred to as the CIDI-Core) covers two of these diagnostic systems, ICD-10 and DSM-III-R for a subset of diagnoses.

The original substance abuse and dependence sections of the CIDI came from the Diagnostic Interview Schedule (DIS; Robins et al, 1981); they assess the abuse of and dependence on tobacco, alcohol, cannabis, barbiturates and tranquillisers, amphetamines, cocaine, heroin and other opiates, phencycidine (PCP) and other hallucinogens and inhalants according to the DSM systems, the Feighner criteria (Feighner et al, 1972), the Renard Diagnostic Interview (RDI; Heizer et al, 1981) and Edwards Gross criteria (Edwards \& Gross, 1976). With new developments in the nomenclature of the DSM and ICD systems, the WHO/ADAMHA Joint Project appointed a task force on alcohol- and drug-related disorders which suggested that the content and format of the substance use sections be revised to accommodate the classification changes. The task force also recommended adding: (a) symptom specific onset and recency questions to assess the natural history of substance abuse; (b) sections on the specific withdrawal symptoms and physical, social and psychological consequences for each drug; and (c) quantity/frequency questions to assess the severity of the dependence syndrome. Questions were developed to cover these areas; however, it was apparent that the application of an 
optimal version would be too detailed to be included in the CIDI-Core where a substance use disorder was just one among many psychiatric disorders covered, so this lengthier version was kept as a separate instrument - the CIDI Substance Abuse Module (SAM; Robins et al, 1990).

The symptom questions in the SAM were field tested for inter-rater reliability in St Louis and were found to have excellent one-week retest reliability among substance abusers in treatment (Cottler et al, 1991). Thus, the SAM questions influenced an early version of the CIDI-Core. An international sub committee was formed to suggest further refinements to the CIDI-Core and these changes were then pre tested as part of the 1988 wave I CIDI-Core field trial in 18 sites around the world.

The main purpose of the field trial was to assess the CIDI-Core in a variety of research settings and cultures. The reliability and acceptability of the psychiatric symptom questions and diagnoses, other than those pertaining to substance use disorders, were found to be reliable and acceptable (see previous paper, this issue). The present paper describes the results of the CIDI field trial in terms of the acceptability of the substance questions in various cultures, the inter-rater reliability for these questions, and difficulties in translating relevant words and concept into different languages. Further suggestions for improvements are discussed. In addition, analyses have been done which assess system differences between ICD-10 and DSM-III-R substance abuse and dependence disorders.

\section{Method}

The CIDI-Core field trials, including sample characteristics, are described in detail in the previous paper (this issue, pp. 645-653). Briefly, 590 subjects from 18 centres around the world participated in the first wave, which began in spring 1988. At each site a minimum of 25 persons were interviewed. At six sites the subjects were hospital in-patients; at 11 sites they were hospital out-patients (in Minneapolis there were both in- and out-patients). One site sampled those enrolled with a health maintenance organisation and another recruited patients from a general practice.

The principal investigators from each site underwent a one-week training course in Munich. The original CIDI-Core was written in English. Strict back-translation methods were followed by each centre for translating both interviews and training materials. The principal investigators then trained the 53 clinicians and 55 non clinician interviewers who participated in these trials. Each subject was independently rated by an interviewer and an observer. While the interviewer queried the respondent using the CIDI-Core, the observer silently documented discrepancies, or questionable areas for clarification with the respondent at the end of the interview.

In developing the section on substance use, care was taken to minimise the confrontational nature of questions on drug abuse. For example, questions on lifetime tobacco use were placed near the beginning of the psychiatric interview to provide an easy introduction, since this behaviour is common, freely admitted, and is not considered threatening by respondents. In contrast, the alcohol and drug sections were placed nearer the end of the interview, after the sections on somatisation, affective, and anxiety disorders, so they could be asked after rapport had been achieved.

'Skip-outs' were provided appropriately; for example, questions about tobacco use were terminated if a respondent reported never smoking daily for a month or more. The questions 
about alcohol were skipped for respondents who denied ever having had a drink and the drug questions skipped if no drug had ever been used six or more times.

Two interview documents per subject were produced - one recorded by the interviewer and one by the observer. Only the interviewer's coding of the responses is used for the descriptive analyses, while for reliability statistics, the observer's record of responses is compared with the interviewer's. The kappa statistic was used to analyse the agreement between raters on all responses which are dichotomous. Diagnostic algorithms developed at Washington University in St Louis were used to determine DSM-III-R and ICD-10 psychoactive substance use diagnoses.

The length of the substance abuse section prohibits the publication of these questions here; however, the questionnaire can be obtained by writing to the authors. The substance abuse sections differed from other sections in that the age of onset and recency of each symptom is ascertained. In other sections, a summary age of onset and recency is ascertained for the first and last time any symptom from a group of symptoms occurred.

\section{Results}

Nine of the 590 subjects interviewed had data missing from the substance abuse section, and so the analyses below relate to 581 subjects.

\section{Acceptability}

The most common complaint was that the substance abuse sections were still unbearably long within the context of a full psychiatric diagnostic interview. The onset questions for individual positive symptoms added considerably to the interview administration time. This complaint was heard from every site and documented in the ratings completed by each interviewer at the end of the interview session. The acceptance ratings of the alcohol, drug and tobacco section are shown in Table1.The tobacco section received scores indicative of overall acceptance, although several sites - in the Netherlands, Australia, Portugal and Italy questioned the inclusion of tobacco use in a psychiatric interview. The alcohol section, although shorter than the drug section, was perceived to be slightly longer than the drug section. This was perhaps because it had to be asked of many more persons, both because alcohol use is more common than drug use and because the alcohol section did not allow a skip for persons whose experience with alcohol was minimal: only respondents who volunteered that they had never had a drink were excused. A unanimous decision was later reached to exclude persons with minimal drinking histories. The revised CIDI-Core now allows a skip for persons who have never had "as many as 12 alcoholic drinks in any one year" and persons who have never had "at least 12 drinks in their lifetime".

None of the subjects who had not drunk as regularly as once a month or had never had four drinks in four hours met diagnostic criteria. More than a quarter (28\%) of the sample could have skipped the section on the basis of these two questions without losing a single case of abuse or dependence. Another $6 \%$ of the sample could have skipped the section after a brief set of questions asking about drinking heavily (having seven drinks once a week for several months), drinking more than intended to, being preoccupied with drinking, giving up activities for drink and having physical, psychological or social problems from drinking. These data suggest that an appropriate quantity/frequency question may function as an efficient screen. 
Other changes to shorten the interview included reducing the number of questions asked to assess a single alcohol criterion, combining recency and onset questions for criteria assessed by multiple questions, and reducing the number of drug categories by combining tranquillisers with barbiturates in a sedative section and heroin with other opiates for an opiate category.

Although the length of the substance abuse section was a problem at all sites, other problems were site specific. These were not unexpected, since cultures differ in attitudes towards substance use and in defining when use becomes abuse. For example, collaborators experienced a number of difficulties with the operationalisation of substance abuse and dependence criteria. One centre noted that binge drinking (staying drunk for two days or longer) is acceptable behaviour during 'official festivals'. To make this question culturally appropriate, exclusionary criteria were added for binges restricted to these occasions. Several sites reported that some respondents were offended by being asked about legal and illegal drugs in the same question and recommended a distinction between them in the interview so that persons who used prescribed medications could admit this without the stigma attached to illicit drug use. Thus, separate categories were provided for prescribed and illicit drugs.

Interviewers, respondents and translators in some sites noted problems with the translation of certain words in the interview. For example, the word 'drink' frequently used as a noun and a verb) was not clear to all respondents, since it does not mean an 'alcoholic beverage' in some cultures, and in others implies whisky but not beer or wine. Measuring intake was problematic because there are cultures which have no standard 'drink' in terms of alcohol content and strength. Additionally, the words 'high', tolerant' and 'dependent' used throughout the interview, are either not translatable or difficult to translate into some languages.

Many of the differences noted are unavoidable for substance disorders, which can arise as a consequence to a variety of substances. Thus, the Joint Project Task Force recommended that investigators should know their local customs surrounding drinking and drug use including the usual volume drunk and the alcohol content by volume, and that translations be faithful to the interview's intent rather than to its original wording. The task force also suggested that the interviewers be well trained on the intent of all questions.

\section{Rates of drug use and symptoms}

In spite of cultural differences, there was an amazing similarity across sites in the types of drugs used and symptoms reported. All centres asked the tobacco, alcohol and drug questions in the CIDI-Core. In all sites except Beijing, at least one respondent reported using drugs at some time in his/her life. Most subjects reported smoking daily for a month or more at some time in their life (56.3\%). Over a quarter (25.1\%) of the sample met criteria for lifetime heavy drinking (defined as ever drinking the equivalent of 20 drinks of liquor in one day, daily drinking of at least seven drinks for two weeks, or two months of drinking at least seven drinks a week). A quarter of the entire sample $(n=147)$ reported never drinking; these persons are excluded from the remainder of the alcohol analyses. Illicit use of drugs six or more times was reported by only $14 \%$ of the sample. Marijuana was the most commonly used drug (12\%), followed by tranquillisers, barbiturates and amphetamines (each 5\%). Cocaine use was reported by $4 \%$ of the sample, hallucinogens and opioids were less commonly reported (3\% each). Inhalants and PCP were the least commonly used (1\% each).

Common symptoms relating to substance use are shown in Table 2. Experiencing emotional problems from drugs, developing tolerance to drugs, and using larger amounts of drugs than intended were each reported by nearly half the persons reporting any use of drugs six or more 
times. The use of the same criteria for abuse and dependence for all substances allows us to report the proportion positive for these symptoms among all drinkers and among those who had been heavy drinkers (Table2). Among both types of drinkers, social problems and tolerance were the most common symptoms reported. Heavy drinkers were at least twice as likely to report experiencing any symptom.

\section{Reliability}

An additional purpose of the field trial was the evaluation of the reliability of diagnoses and of the specific questions used to make alcohol and drug abuse and dependence diagnoses. Kappa values in excess of 0.93 were obtained for agreement between observer and interviewer reports of lifetime use of these substances and over 0.94 for each of the symptoms in Table 2, indicating excellent reliability.

\section{Nosological comparisons}

Although not one of the goals of this field trial, these data offer an early opportunity to address differences in the way in which the alcohol and drug disorders are classified in DSMIII-R and ICD-10. Comparisons are provided for the most commonly reported substances: alcohol, marijuana, opioids, and stimulant use. The comparisons exclude tobacco disorders because DSM-III-R and ICD-10 criteria were not assessed in the CIDI-Core version used in the field trials. In Table 3, the number of persons who met DSM-III lifetime criteria is crosstabulated with the number who met lifetime criteria for an ICD-10 diagnosis. Thus, for ICD10 , three or more symptoms are necessary, but the requirement for these symptoms to occur together in one month or in previous 12 months is ignored.

The comparisons for alcohol exclude the 147personswho never had a drink. The comparisons for the remaining428 persons show that DSM-III-R has labelled 104 persons as dependent while ICD-10 has so labelled only 94. More persons (48) met criteria for ICD-10 'harmful use' than met criteria for DSM-III-R 'abuse' (35).Of the 35 persons in the DSM-III-R abuse category, 11were in the ICD-10 harmful use category, while 8 of the 94 labelled dependent in ICD-10 were labelled abusers according to DSM-III-R. The ICD-10 harmful use category has broader limits than the DSM-III-R abuse category. The kappa statistic for overall agreement for all alcohol disorder categories is in the good to excellent range (0.81).

No respondent met ICD-10 criteria for marijuana, stimulant or opiate dependence, although 17 persons were diagnosed with marijuana dependence and 12 diagnosed with stimulant dependence according to DSM-III-R criteria. However, for marijuana, as with alcohol, more persons met criteria for ICD-10 harmful use than for DSM-III-R abuse.

The low kappa value for marijuana (0.48) indicates that diagnostic agreement between the two systems was not as good as for alcohol. The poor agreement $(\mathrm{kappa}=0.46)$ for stimulants overall was due primarily to the lack of agreement for the dependence category. Opioid disorders were rare among persons who used opioids; furthermore, dependence criteria were not met for either of the diagnostic systems. The two systems' distributions of opioid abuse and harmful use were approximately equivalent (kappa=0.82). The higher agreement for opioids (compared with other drugs) was probably due to the more severe consequences of these substances. At least for three commonly reported substances - alcohol, marijuana and stimulants - the CIDI Core questions which cover the DSM-III-R system seem to cast a wider net for dependence than the questions which cover the ICD-10 system. 


\section{Discussion}

The substance use sections of the CIDI-Core were the sections most extensively revised as a result of the field trial. These revisions have reduced the length of the instrument and recognised cultural differences in patterns of substance use and language. Reliability presented no problem. Furthermore, because the criteria continue to evolve, a symbiotic relationship exists between criteria development and instrumentation, which guarantees continued change. As Robins (1989) and Cottler \& Keating (1990) have demonstrated, this relationship is particularly strong with substance abuse and dependence because a lack of clarity in diagnostic concepts is sometimes only realised once the operationalisation of these concepts is attempted. The difficulty encountered is intensified when the questions and concepts must be translated into a variety of languages and understood by persons from many cultures.

In spite of the difficulties, the CIDI-Core substance use sections, as they appeared during our first field effort, have been found to be acceptable and highly reliable. The field trial confirmed the authors' suspicions that the sections were too long. Incorporating a skip instruction early in the alcohol section should help solve the problem. Moreover, as with other diagnostic sections of the CIDI-Core, revisions have been instituted by the task force to make the interview shorter and more palatable.

Even though the CIDI-Core has been revised, we have no reason to believe that the reliability of these new, revised questions will be poorer than that reported for these original questions, since the changes were in response to problem areas and the changes were well thought out, and pre-tested in certain instances. However, it must be emphasised that the newest changes have not undergone rigorous pilot testing. The plans for a further set of international studies which assess test-retest reliability and diagnostic validity are underway now, especially focused on alcohol and drug disorders.

The investigators were not surprised to find excellent reliability with the substance abuse sections since the other diagnostic sections were equally reliable and many of the same questions had been found to have excellent one-week test-retest reliability in a population of substance abusers in St Louis (Cottler et al, 1989). But, additionally, because the interviewers and observers were paired together in the same room, the field trial methods may have been biased in favour of good reliability. However, this cost-efficient interviewer/observer design was chosen instead of the more costly test-retest method. Although not unexpected, the favourable results obtained are welcome, especially given the hetero geneous samples and different cultures studied.

The ability to compare diagnostic systems on an international level is one of the most promising and fascinating aspects of the CIDI-Core and the WHO/ADAMHA Joint Project. Our early nosological comparisons indicate either that DSM-III-R's dependence criteria are broader than ICD-10's, or perhaps that our questions which cover the ICD-b need improvement. Perhaps for alcohol, stimulants and marijuana, the diagnostic assignments of 'caseness' are different because the DSM-III-R concept of dependence includes social impairment, which ICD-10's does not. However, these early comparisons should not be interpreted as a failure of the systems to agree but as an indication of the type of analyses which can be performed on data collected by a structured instrument of this type. We must remember that the ICD system has been in flux since these questions were written and the DSM system is in the process of being revised. These changes in criteria will necessitate further changes in CIDI questions, which will lead to further pre-testing. 
These important comparisons should be replicated by other investigators and with larger samples. Additionally, work needs to be done to evaluate the nomenclatures, and the differences and similarities between the classification systems for other categories of substances. These findings add to the preliminary findings from a study of drug abusers in St Louis which revealed that for many psychoactive substances, DSM-III-R labels a larger proportion of users as dependent than the earlier DSM-III system (Cottler et al,1989).

The difficult task is behind us. Producing an instrument which can be used comfortably and reliably in so many different cultures is a tremendous achievement for psychiatric epidemiology and clinical psychiatry. Investigators from all over the world can now use the CIDI family of instruments to measure the problems associated with drug and alcohol use reliably and most importantly to make communication of substance abuse problems between many nations possible.

\section{Acknowledgements}

The authors wish to thank the members of the WHO/ADAMHA Task Force on Psychiatric Assessment Instruments. Dr Cottler acknowledges the support of DA05585, DA05619, DA06163, a MacArthur Foundation DSM-IV Analysis Award, and the technical assistance of Jeanette Haynes.
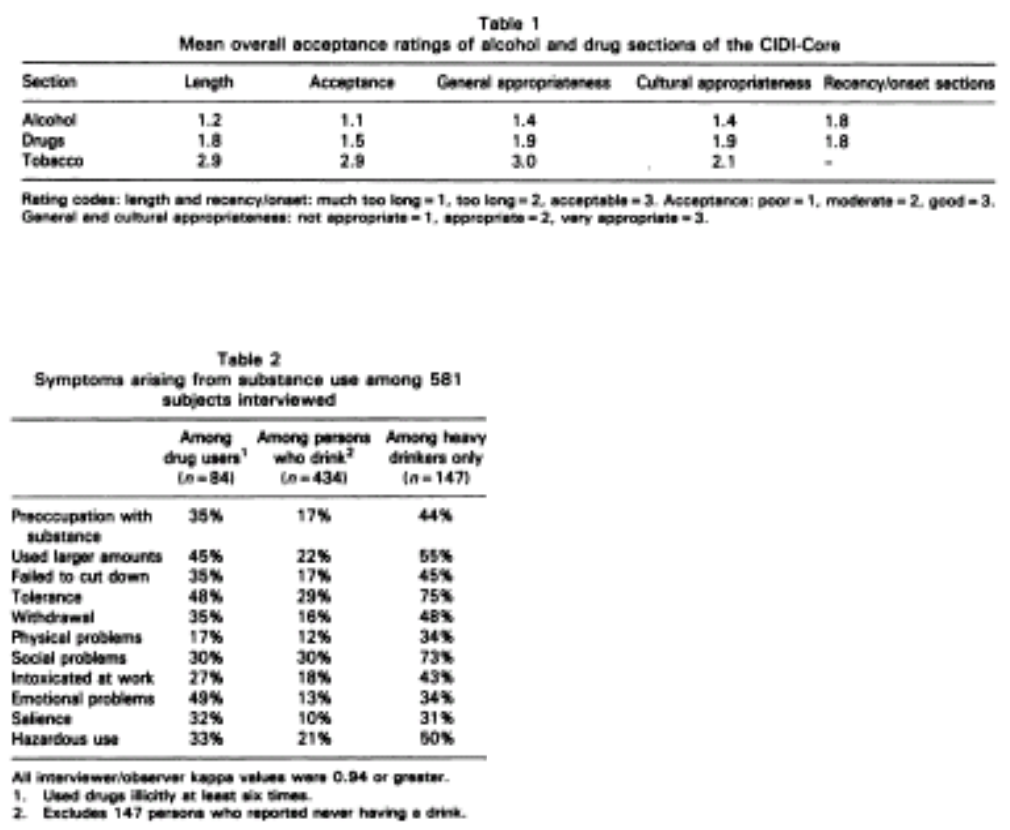


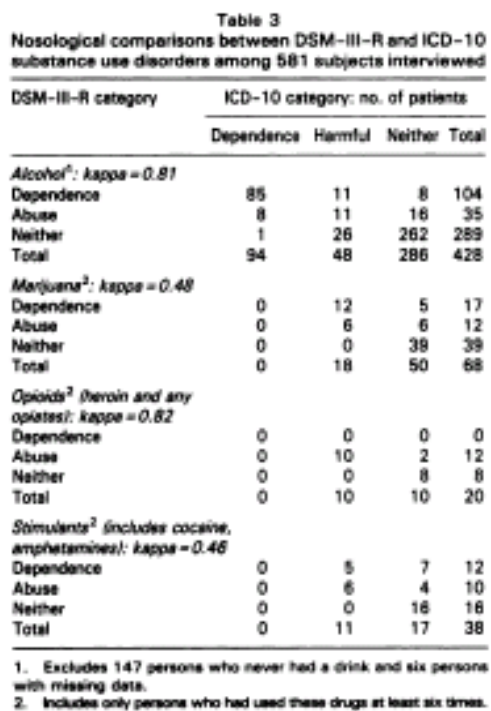

\section{References}

AMERICAN PSYCHIATRIC ASSOCIATION (1980) Diagnostic and Statistical Manual of Mental Disorders (3rd edn) (DSM-III). Washington, DC: APA.

-- (1987) Diagnostic and Statistical Manual of Mental Disorders (3rd edn, revised) (DSM-III-R). Washington, DC: APA.

BURKE, J. D. (1986) Diagnostic categorization by the Diagnostic Interview Schedule (DIS): a comparison with other methods of assessment. In Mental Disorders in the Community. Findings from Psychiatric Epidemiology (eds 3. Barret \& R. M. Rose), pp. 255-285. NewYork: Guilford Press. COTTLER, L. B., ROBINS, L. N. \& HELZER, J. E. (1989) The reliability of the CIDI-SAM: a comprehensive substance abuse interview. British Journal of Addiction, 84, 810-814.

-- \& KEATING, S. (1990) Operationalization of alcohol and drug dependence criteria by means of a structured interview. In Recent Developments in Alcoholism (ed. M. Gallantes). New York: Plenum. --, HELZER, J. E. \& MAGER, D., et al (1991) Diagnostic agreement between DSM-III and DSM-III$\mathrm{R}$ substance abuse disorders. Alcohol Abuse and Dependence (in press).

EDWARDS, G. \& GROSS, M. M. (1976) Alcohol dependence: provisional description of a clinical syndrome. British Medical Journal, i, 1058-1061.

FARMER, A. E., KATZ, R., MCGIFFIN, P., et al (1987) A comparison between the Present State Examination and the Composite International Diagnostic Interviews. Archives of General Psychiatry, 44, 1064-1068.

FEIGHNER, J. P., ROBINS, E., GUZE, S. B., et al (1972) Diagnostic criteria for use in psychiatric research. Archives of General Psychiatry, 26, 57-63.

HELZER, J. E., ROBINS, L. N., CROUGHAN, J. L., et al (1981) The Renard Diagnostic Interview: its reliability and procedural validity with physicians and lay interviewers. Archives of General Psychiatry, 38, 393-398.

ROBINS, L. N. (1989) Diagnostic grammar and assessment: translating criteria into questions. The Validity of Diagnosis (eds L. Robins \& J. Barrett). New York: Raven Press.

--, HELZER, J. E., CROUGHAN, J., et al (1981) The National Institute of Mental Health Diagnostic Interview Schedule. Its history, characteristics, and validity. Archives of General Psychiatry, 38, 381389.

--, WING, J., WITTCHEN, H.-U., et al (1988) The Composite International Diagnostic Interview: an epidemiologic instrument suitable for use in conjunction with different diagnostic systems and in different cultures. Archives of General Psychiatry, 45, 1069-1077.

--, COTTLER, L. B. \& BABOR, T. (1990) The CIDI Substance Abuse Module. St Louis: Washington University.

SEMLER, G. (1990) Reliabilität und Validität des Composite International Diagnostic Interview (CIDI). Regensburg: Roderer Verlag. 
--, WITTCHEN, H.-U., JOSCHKE, K., et al (1987) Test-retest reliability of a standardized psychiatric interview (DIS/CIDI). European Archives of Psychiatry and the Neurological Sciences, 236, 214-222. --, -- \& ZAUDIG, M. (1988) The test-retest reliability of the German version of the Composite International Diagnostic Interview on RDC diagnoses and symptom level. International Classification in Psychiatry, Unity and Diversity (eds J. E. Mezziah \& M. von Cranach), pp. 221-234.London: Cambridge University Press.

WING, J. K., COOPER, J. E. \& SARTORIUS, N. (1974) The Description and Classification of Psychiatric Symptoms. An Instruction Manual for the PSE and CATEGO System. London: Cambridge University Press.

WITTCHEN, H.-U., BURKE, J. D., SEMLER, G., et al (1989) Recall and dating reliability of psychiatric interview (CIDI/DIS). Archives of General Psychiatry, 46, 437-443.

--, ROBINS, L. N., COTTLER, L., et al (1990) Interrater reliability of the Composite International Diagnostic Interview (CIDI). Results from the multicenter WHO/ADAMHA field trials (wave I). In Psychiatry - A World Perspective, vol. 1 (ed. C. N. Stefanis), pp. 125-132. International Congress Series Vol. 900. Amsterdam: Elsevier.

WORLD HEALTH ORGANIZATION (1987) Composite International Diagnostic Interview, Core Version 0.0. Geneva: WHO.

-- (1989a) ICD-10, Chapter V, Mental and Behavioral Disorders (including disorders of psychological development) - Diagnostic Criteria for Research. Draft for field trials. Geneva: WHO.

-- (1989b) Composite International Diagnostic Interview. Geneva: WHO. 